\title{
Applications of the COG Multiparticle Monte Carlo Transport Code to Simulated Imaging of Complex Objects
}

\author{
Richard M. Buck \\ James M. Hall
}

This paper was prepared for submittal to the

SPIE's 44th Annual Meeting of the International Symposium on

Optical Science, Engineering, and Instrumentation

Denver, $\mathrm{CO}$

July 18-23, 1999

June 1999

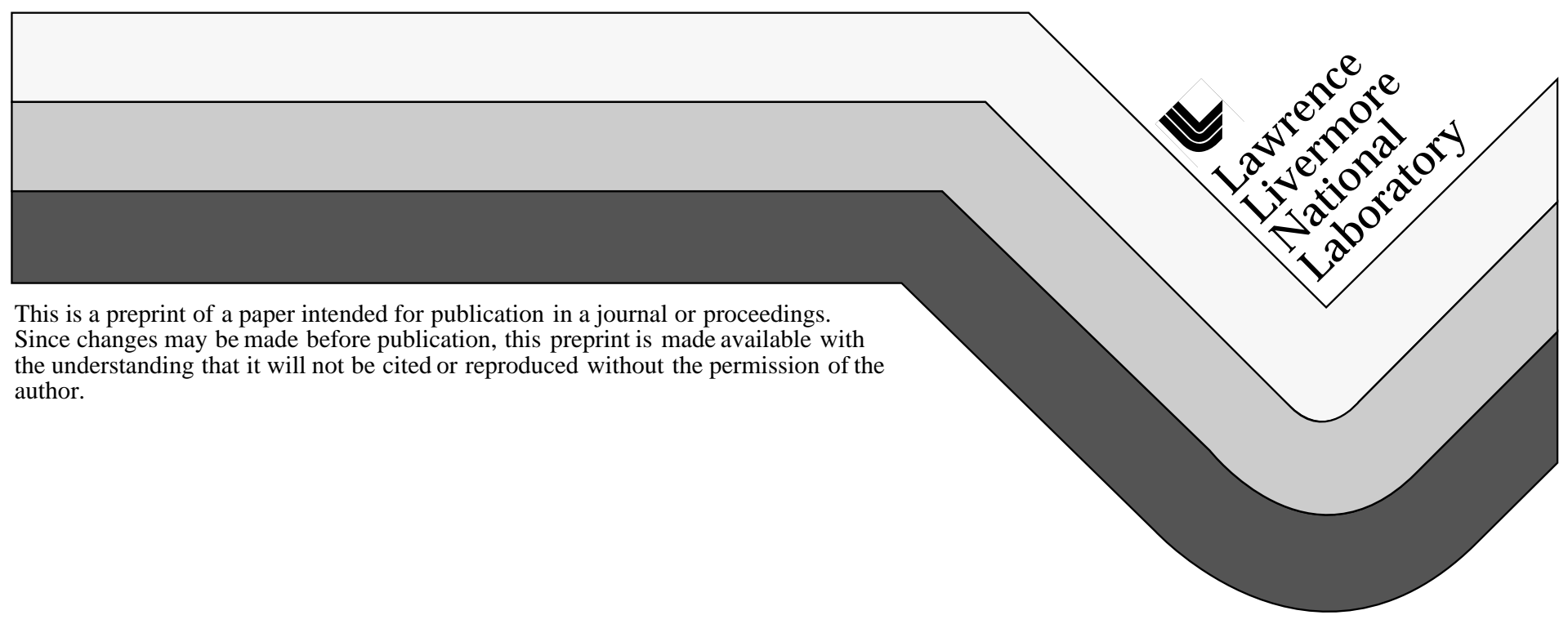




\section{DISCLAIMER}

This document was prepared as an account of work sponsored by an agency of the United States Government. Neither the United States Government nor the University of California nor any of their employees, makes any warranty, express or implied, or assumes any legal liability or responsibility for the accuracy, completeness, or usefulness of any information, apparatus, product, or process

disclosed, or represents that its use would not infringe privately owned rights. Reference herein to any specific commercial product, process, or service by trade name, trademark, manufacturer, or otherwise, does not necessarily constitute or imply its endorsement, recommendation, or favoring by the United States Government or the University of California. The views and opinions of authors expressed herein do not necessarily state or reflect those of the United States Government or the University of California, and shall not be used for advertising or product endorsement purposes. 


\title{
Applications of the COG multiparticle Monte Carlo transport code to simulated imaging of complex objects
}

\author{
Richard M. Buck and James M. Hall* \\ Lawrence Livermore National Laboratory \\ P. O. Box 808, MS L-59, Livermore, CA 94550
}

\begin{abstract}
COG is a major multiparticle simulation code in the LLNL Monte Carlo radiation transport toolkit. It was designed to solve deep-penetration radiation shielding problems in arbitrarily complex 3D geometries, involving coupled transport of photons, neutrons, and electrons. COG was written to provide as much accuracy as the underlying cross-sections will allow, and has a number of variance-reduction features to speed computations. Recently COG has been applied to the simulation of highresolution radiographs of complex objects and the evaluation of contraband detection schemes. In this paper we will give a brief description of the capabilities of the COG transport code and show several examples of neutron and gamma-ray imaging simulations.
\end{abstract}

Keywords: Monte Carlo, radiation transport, simulated radiography, nonintrusive inspection, neutron imaging.

\section{INTRODUCTION}

$\mathrm{COG}^{1}$ is a Monte Carlo radiation transport code designed primarily to solve coupled neutron-photon-electron deep penetration (shielding) problems in complex three-dimensional geometries. In addition, COG can calculate the criticality of assemblies of fissile materials, and solve problems involving activation of neutron-irradiated materials. The recently-added capability of energetic proton transport enables the evaluation of experimental designs in projects involving particle accelerators, such as proton radiography and accelerator-driven transmutation of nuclear-fission wastes.

In the last few years, COG has been exploited as a tool for evaluating proposed radiographic imaging schemes. We have been able to accurately simulate and analyze the performance of gamma-ray and neutron contraband detection systems, which have been proposed for scanning airline checked baggage, carry-on luggage, and cargo containers. Based on our experience, we are convinced that detailed numerical modeling provides a much more accurate estimate of the actual performance of complex experimental systems than does simple analytical modeling. Additionally, building a numerical model allows the experimenters to quickly explore the full parameter space available to them before committing their ideas to hardware. This approach has frequently led to improvements in experimental designs at LLNL and reductions in overall system development costs. A key strength of COG simulations is the ability to separate out and analyze in detail complex secondary effects (such as scattering, room return, secondary particle production) which are often neglected in preliminary design calculations and are experimentally difficult and/or expensive to measure. As we shall see in some later examples, these effects can often mask the desired signals and considerably degrade detection performance.

\section{THE MONTE CARLO TRANSPORT METHOD}

A Monte Carlo code like COG solves the Boltzmann transport equations by simulating as exactly as possible the trajectories of particles emitted by a radiation source. Pseudo-random numbers are used to sample the source distribution in position, energy, and angle. These particles are then traced into the user's geometry and scored at one or more points of interest, if they reach them (Figure 1). Particles can scatter, be absorbed, or react with atoms and nuclei to generate new secondary particles to be tracked. Each collision outcome is determined by using pseudo-random numbers to choose a reaction and to sample secondary particle distributions for particle type, energy, and angle. A particle history is the sequence of events from a particle's birth at the source, through its interactions in the user's geometry, to its termination (by absorption or leakage from the problem). The result of a COG run is just a collection of independent particle histories, which are analyzed to determine the flux at specified locations (called "detectors"). In a radiographic simulation, each pixel in the image is a detector, so a $1000 \times 1000$ pixel image requires $10^{6}$ detectors.

\footnotetext{
*Correspondence: buck2@1lnl.gov; hall9@1lnl.gov
}

Monte Carlo codes can only give statistical answers. If a problem is rerun using a different set of random numbers, numerically different answers will be obtained. But if enough source particles have been run (so that the problem has not been undersampled), then the two results are very likely to lie within one or two standard deviations of each other. A rough 
estimate of the number of particles needed to achieve a desired statistical uncertainty in a detector score can be calculated as follows. For Poisson statistics, the estimated standard deviation of a score of $\mathrm{N}$ particles is $\sqrt{ } \mathrm{N}$. If we want the relative error to be $1 \%$, then $\mathrm{N}$ must be about $10^{4}$. For a single detector, this is readily achieved by a short COG calculation. But if we wish to achieve this relative error in a radiographic simulation using a $1000 \mathrm{X} 1000$ pixel image, we will need of order $10^{10}$ scoring particles. This is now a huge problem, and can only be solved in a reasonable time by utilizing many CPUs in a parallel computation.

\section{COG DESIGN FEATURES}

COG was expressly designed to solve deep-penetration problems typified by such examples as contraband detection schemes for large-containers, and radiography of moderately opaque objects. These problems are characterized by having many meanfree-paths of scattering materials between source and detector, a complex geometry, and a large number of possible materials. Such problems are beyond the scope of deterministic transport calculations, and also cannot be solved by simple analog Monte Carlo transport computations. COG has several variance-reduction techniques that permit reliable answers to be obtained, even when the radiographed object is many mean-free-paths thick.

COG was written to be free of physics compromises found in some other transport codes. It uses pointwise cross sections and exact angular scattering data to utilize the resolution of the cross section data as fully as possible. COG will produce results as accurate as the underlying cross sections allow. Almost all of the code physics resides in the numerical databases, which allow for easy upgrading of capabilities when better cross section evaluations become available. COG can use either the LLNL Evaluated Neutron Data Library (ENDL) database or Brookhaven National Laboratory's Evaluated Nuclear Data File (ENDF) databases for neutron transport, and the LLNL Evaluated Photon Data Library (EPDL) photon library for photon transport. The use of detailed, evaluated nuclear and atomic interaction databases such as these allows users to account for multiple scattering, energy straggling, and secondary particle production phenomena which may significantly affect the performance of an imaging system, but may be difficult or impossible to estimate using simple analytical models. For the transport of electrons and the production of Bremsstrahlung and annihilation radiation, COG uses an electron transport kernel taken from the $\mathrm{EGS}^{2}$ electron transport code. This capability is especially useful for modeling electron-beam x-ray sources, and electron/gamma-ray cascades in irradiated objects. Frequently the Bremsstrahlung emitted by a radiographed target contributes significantly to the background at the image plane, and thus requires a careful calculational evaluation. COG's accuracy and reliability have been proven through extensive benchmarking on radiation shielding and criticality problems studied in recent LLNL programs ${ }^{3}$.

Problem geometries can be set up manually using simple but powerful constructs (more than 20 elementary surface types such as planes, cylinder, spheres, and boxes are available), or rapidly developed using the new COG Graphical User Interface. The COG GUI uses a forms-based display to gather input, present menus of choices, and check for input errors and consistency. Geometry parts can be visualized through cross-section or perspective views to assure their fidelity, and a new feature allows the user to "fly around" his geometry by changing his viewpoint in real time. COG provides a variety of builtin source and detector models, but if the user needs a special source or detector, the user can write his own set of routines which are dynamically linked into COG at run time. This feature permits the user to do complex post-processing of detector scores.

Radiography simulation requires scores at thousands of pixel locations, necessitating the transport of several billion particles to achieve good $\mathrm{S} / \mathrm{N}$ ratios. To accomplish these very large calculations, COG has a robust and efficient parallel-processing implementation which allows it to run concurrently over a network of workstations or on a massively parallel processor (MPP). We have commonly made 500-CPU runs on the IBM-SP MPP to create high-resolution radiographic simulations.

COG versions are available for most popular Unix platforms, the IBM PC, and the Apple Powermac. COG is available to qualified users who enter into a Collaboration Agreement with LLNL.

\section{OVERVIEW OF COG CAPABILITIES}

Figure 1 lists the code's capabilities for representing geometric surfaces, radiation sources, transport aids (variance-reduction procedures), and simulated detectors. Figure 2 lists the neutron, photon, electron, and activation databases which COG uses. Figure 3 gives the kinetic energy limits for transport of each particle type. 


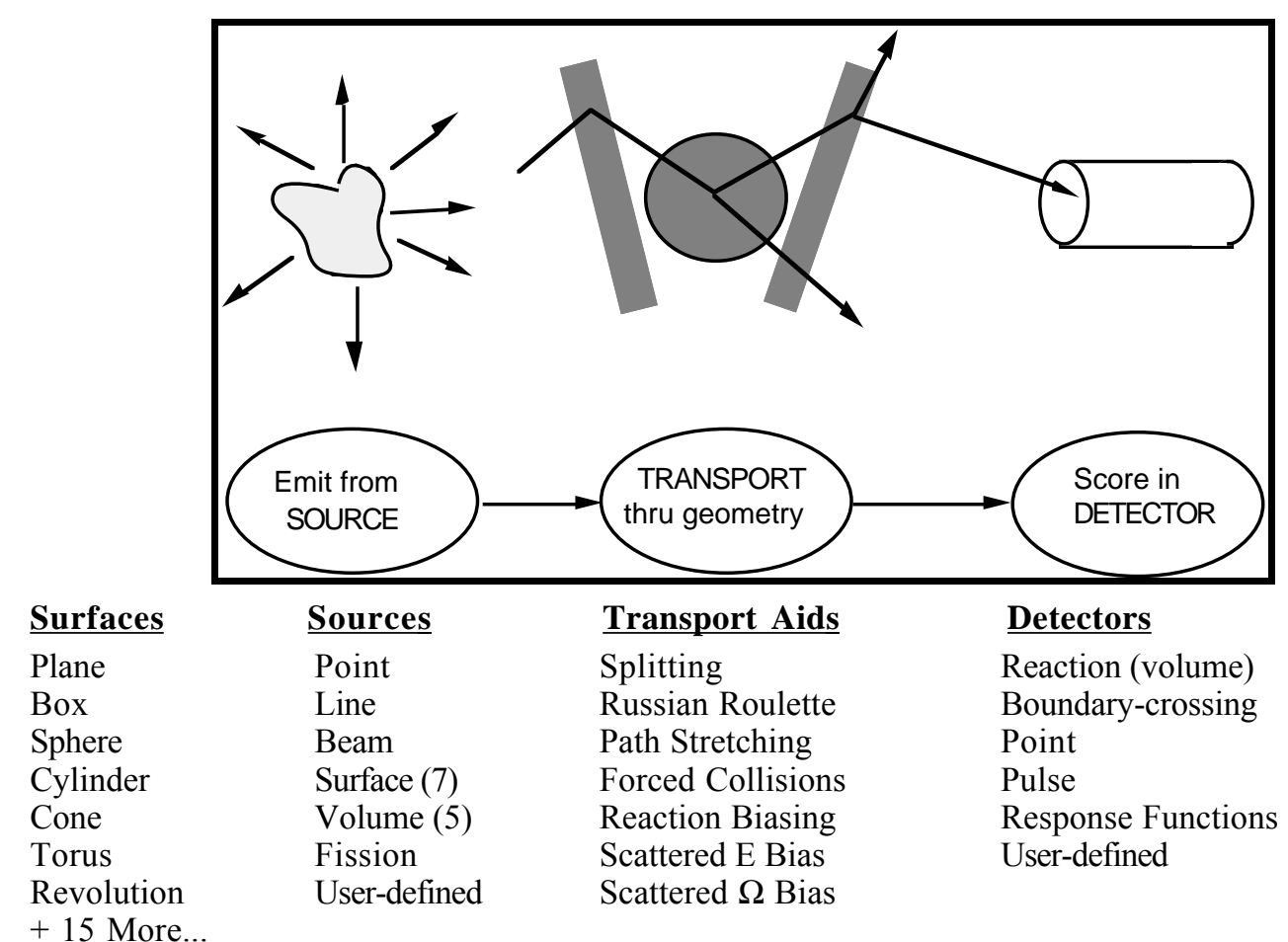

Figure 1. COG capabilities

Neutron Databases

ENDL-90 (LLNL)

ENDF/B-5 (BNL) ${ }^{1}$

ENDF/B-6 (BNL)

\section{Neutrons}

thermal - $30 \mathrm{MeV}$
Photon Database

EPDL (LLNL)

Figure 2. COG databases

Photons

$10 \mathrm{eV}-100 \mathrm{GeV}$

Electrons

$10 \mathrm{keV}-1000 \mathrm{GeV}$
Activation Database

Activation Library

ACTL (LLNL)

Figure 3. Transport energy limits 


\section{COG APPLICATIONS TO SIMULATED RADIOGRAPHY}

\subsection{Contraband detection}

A variety of nonintrusive inspection systems have been proposed over the past several years for the detection of hidden contraband in airline luggage and shipping containers. The majority of these proposed techniques depend on the interaction of radiation with matter to produce signatures specific to the contraband of interest, whether illegal drugs or explosives. Almost without exception, these schemes have been based on simplified physical models which assume straight-line radiation transport and single interactions between the probe particles and the container of interest, assumptions which we have found rarely prove true in the world outside of the laboratory.

\subsubsection{Nonintrusive luggage scanning systems}

In an effort to assess the utility of different types of radiation sources in these systems, we have recently used COG to simulate a potential airport luggage scanning system. Figure 4.1 shows the simulated image of a particularly nefarious (and hopefully fictitious!) overnight bag irradiated by $100 \mathrm{keV}$ gamma rays (resolution $\approx 2.5 \mathrm{~mm}$ ). This image is the result of a $100 \mathrm{M}$ particle run executed in parallel on three different platforms. Figure 4.2 shows a simulated image of the bag when alternatively irradiated by $638 \mathrm{keV}$ neutrons. Notice that different objects stand out in the two radiographs; photons are more highly absorbed by high- $Z$ materials (gun and bullets), whereas low-Z materials (explosive and cocaine) more strongly scatter neutrons. This illustrates the well-known principle that multi-band or multiparticle detection schemes gather much more discriminating information about material composition than any single-energy scan.

But it is also important to note that Fig. 4.2 shows only the contributions from the unscattered neutron flux. The power of a COG simulation lies in its ability to accurately calculate scattering, straggling, and secondary particle effects which constitute the unwanted background at the image plane. Figure 4.3 shows the image formed by just the scattered neutrons. Figure 4.4 shows the total (direct plus scattered) neutron image. In this image, the strong signal from the explosive and cocaine is considerably weakened, and the gun image faintly discernible in the direct image has been completely washed out by background. Figure 4.5 compares lineouts taken through the explosive for direct, scattered, and direct + scattered images. Note that the strong flux drop-out feature in the direct image, which corresponds to the explosive package, is nearly completely filled in by scattered flux. This illustrates the point that proposed imaging schemes which appear promising in simple hand or analytic calculations need to be assessed by a code which can account for scattering to all orders.

\section{Terrorist Overnight Bag (100 keV $\gamma ; 2.5 \mathrm{~mm}$ resolution)}

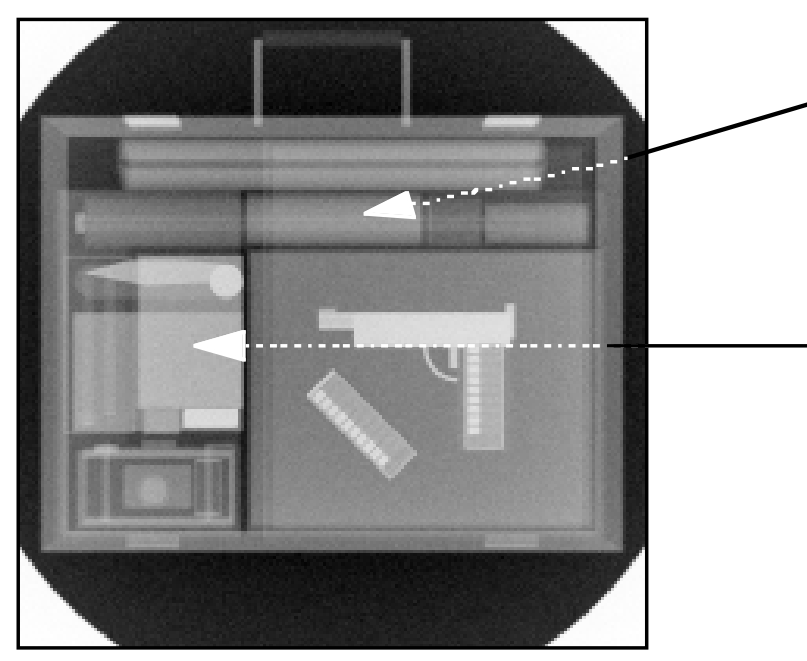

\section{Contents:}

- newspaper

- sugar container

- cocaine stash

- travel umbrella

- switchblade knife

- paperback book

- plastic explosive

- pen and pencil set

- electronic camera

- automatic pistol

- various clothing

- flat notebook
Figure 4.1: This figure shows the COG image of one particularly nefarious overnight bag simulated during our study of airport luggage scanning systems. The bag itself is a standard aluminum shell $(\approx$ $40 \times 30 \times 10 \mathrm{~cm}$ ) with a wood handle, thick cloth covering and steel fittings. 


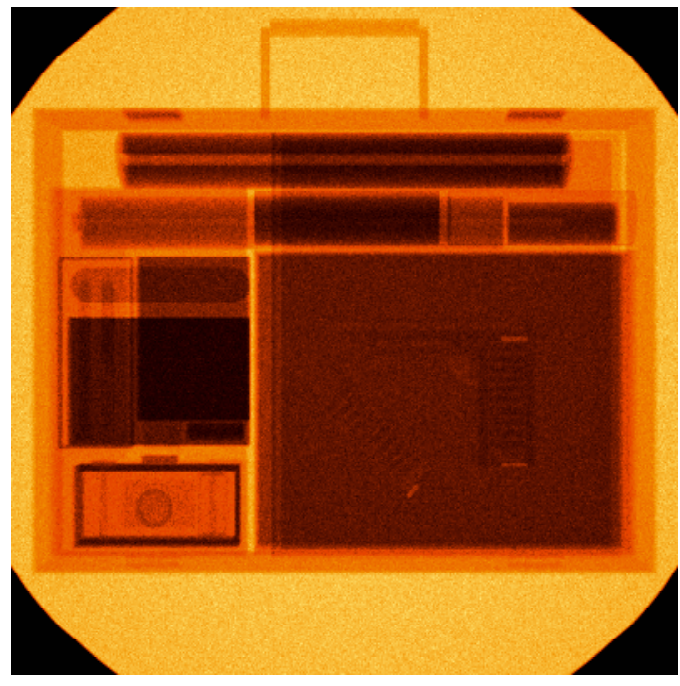

Figure 4.2 COG image of bag formed by simulated irradiation with $638 \mathrm{keV}$ neutrons. Only direct (unscattered) neutrons contribute. Low-Z items such as explosive and cocaine are seen as distinct features.

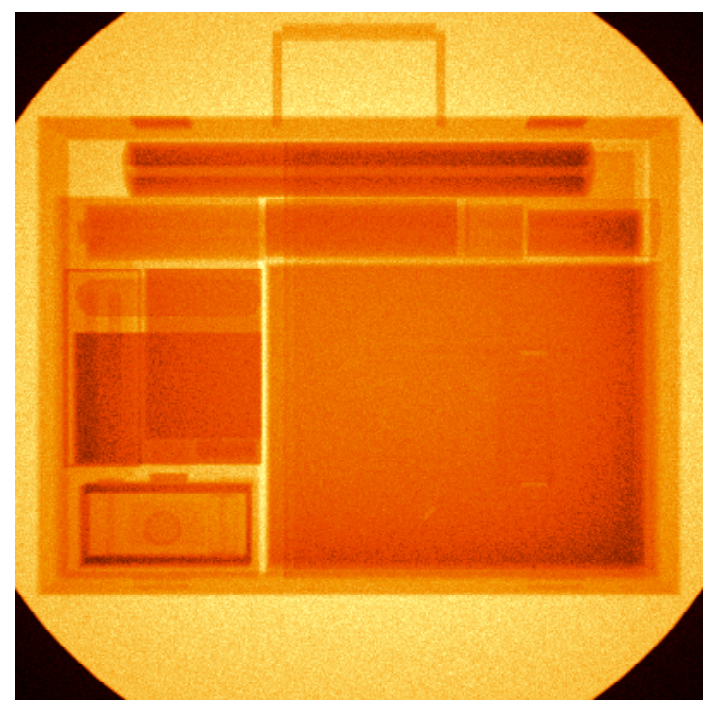

Figure 4.4 COG-computed total neutron image (direct + scattered). Strong features in the direct image are washed out by scattered neutron background.

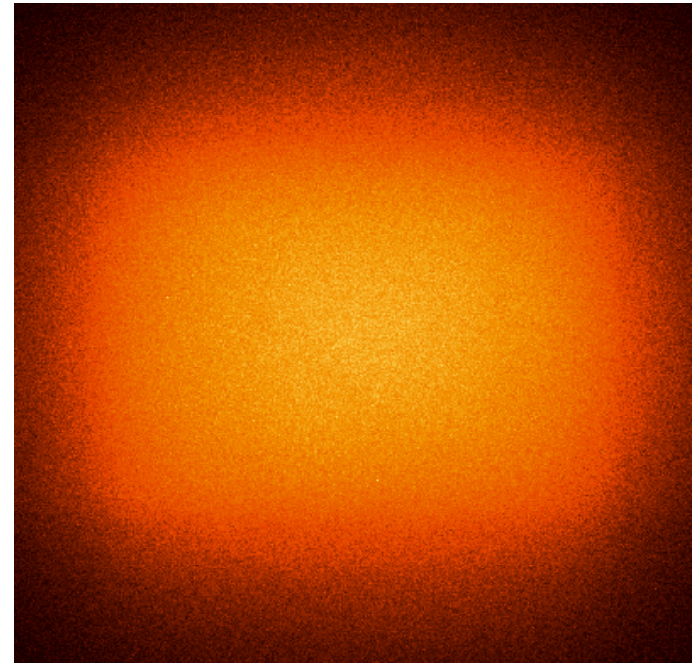

Figure 4.3 COG-computed background at the image plane from scattered neutrons.

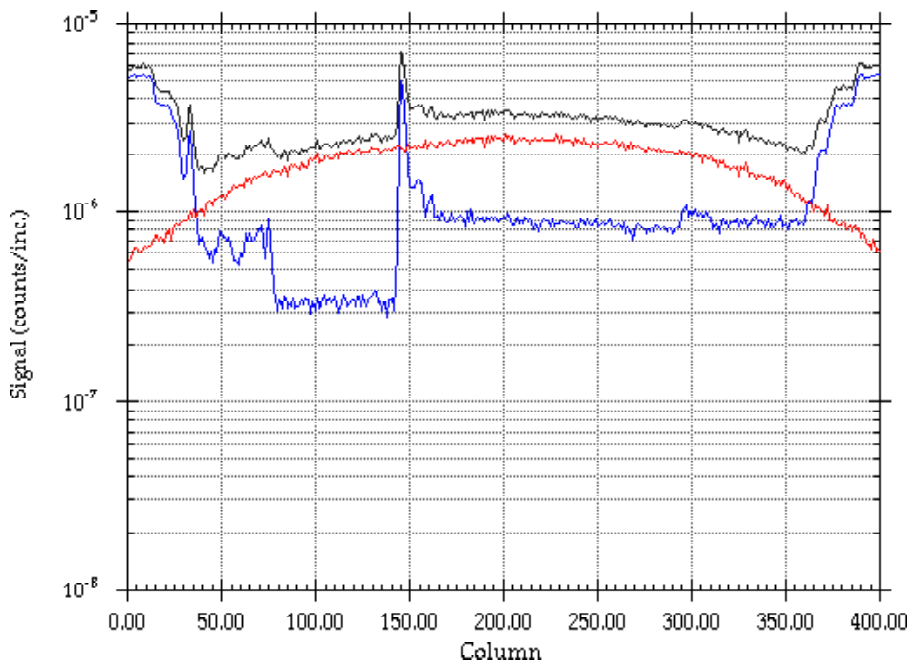

Figure 4.5 Lineouts taken through the explosive and pistol, for the direct, scattered, and direct + scattered image. Note that the strong flux drop-out feature marking the explosive package is almost completely filled in by scattered neutrons. 


\subsubsection{Pulsed fast neutron cargo container inspection system}

COG was used to evaluate a pulsed fast neutron system proposed for rapid inspection of large cargo shipping containers to detect the presence of contraband (including drugs). The scheme used an array of gamma-ray detectors to measure characteristic gamma rays emitted by nuclei excited by a short pulse of $8 \mathrm{MeV}$ neutrons shot into the container side. Computer analysis of the time-resolved detector responses would allow the construction of an image of the scanned section, showing the relative abundances of key elements. To investigate the performance of the proposed system, we modeled a challenging contraband detection scenario: a $25 \mathrm{~cm}$ sphere of cocaine at the center of the cargo container $\left(8^{\prime} \times 8^{\prime}\right.$ in cross section), which was completely surrounded by sucrose, a material with a similar elemental composition. The results of the COG simulation are given in the following figures. Figure 5.1 is a COG analysis plot which shows the primary neutron collision sites in a planar slab of material which contains the beam and the center of the cocaine sphere. Less than $1 \%$ of the beam ever reaches the drug sphere. Figure 5.2 shows a sampling of the scattering events for neutrons of all energies. It is evident that most of the neutrons are thermalized and captured in the sucrose. Figure 5.3 depicts the photon production and scattering locations. The vast majority of these lie in the sucrose, and their signal swamps that from the drug. We concluded that the system would fail to detect the contraband in this case.

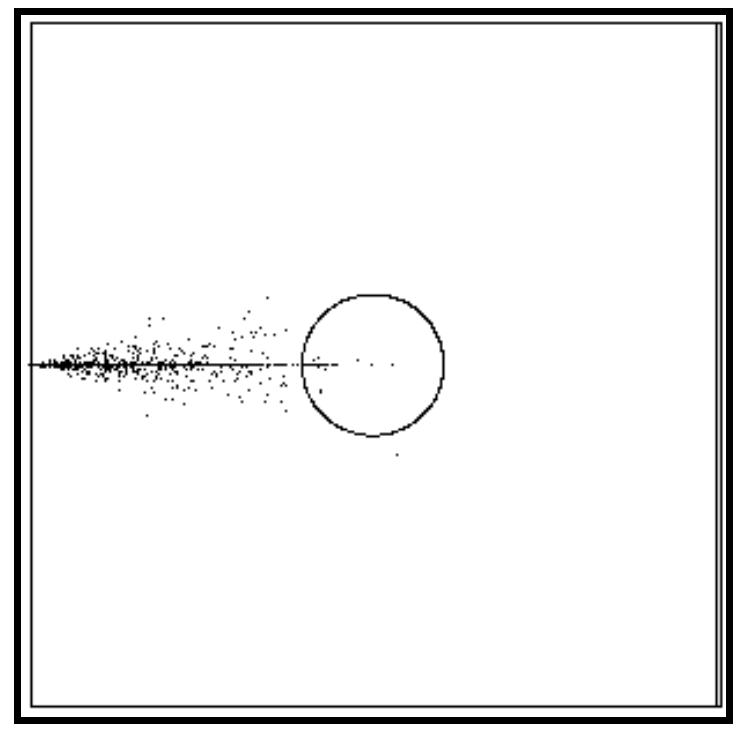

Figure 5.1 Source neutron scattering events

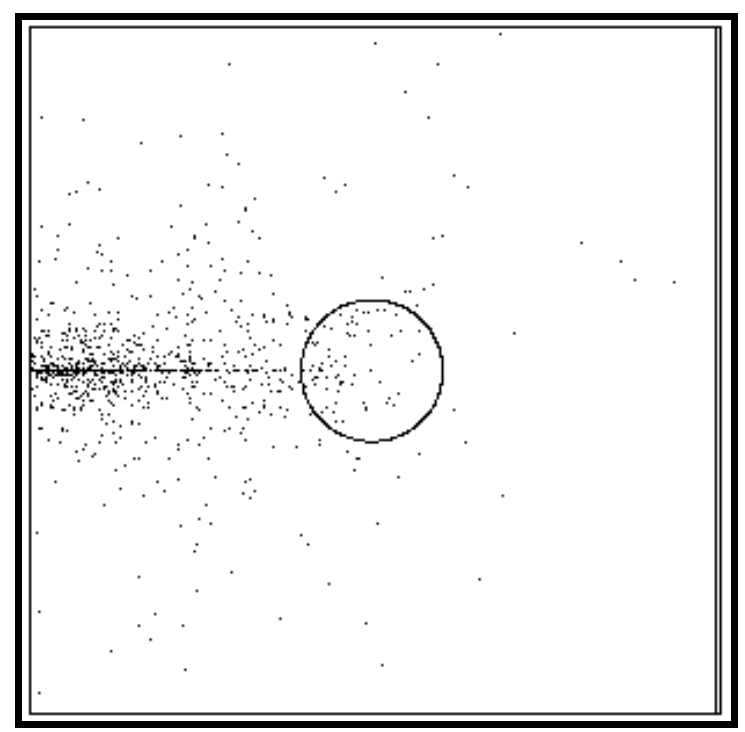

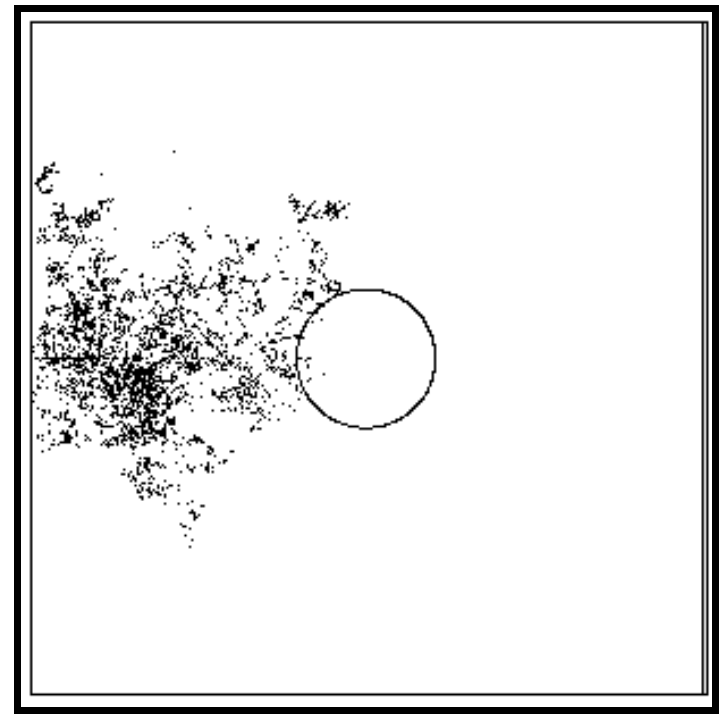

Figure 5.2 Sample of all neutron scattering events

Figure 5.3 Photon production and scattering events for $\mathrm{E}_{\mathrm{p}}>3 \mathrm{MeV}$. 


\subsection{Thick object neutron radiography}

We collaborated in experiments at the Los Alamos Nuclear Science Center (LANSCE) aimed at establishing a proofof-principle for radiography of thick targets using very high energy neutrons $(\approx 40-400 \mathrm{MeV})^{4}$. In those experiments, an assembly consisting of a low-Z disk with a thickness of $2.54 \mathrm{~cm}$ sandwiched between two 5.08 -cm-thick high-Z slabs was used as a phantom target. Several small holes $(4-12 \mathrm{~mm})$ drilled all or part of the way through the disk were used to simulate defects in the low-Z material. A position-sensitive multiwire detector with a heavy metal converter was used to record the neutron image with a spatial resolution of $\approx 1 \mathrm{~mm}$. Since neutron interaction cross sections do not scale strongly with $\mathrm{Z}$, neutron radiography is sensitive to detect defects such as these in low-Z materials even when those materials are heavily shielded by high-Z parts (a difficult task for conventional photon radiography); thus, neutron radiography has the potential to be a powerful nonintrusive inspection tool for thick targets.

During the course of these experiments, we used COG to simulate the result that would be obtained if the LANSCE phantom target were imaged using lower energy $(14 \mathrm{MeV})$ neutrons from a commercially available source. The geometrical model used in the simulation and the resultant image (resolution $\approx 1 \mathrm{~mm}$ ) are shown in Figure 6 . This image is the result of a $100 \mathrm{M}$ particle run executed in parallel on three different platforms

Geometrical model

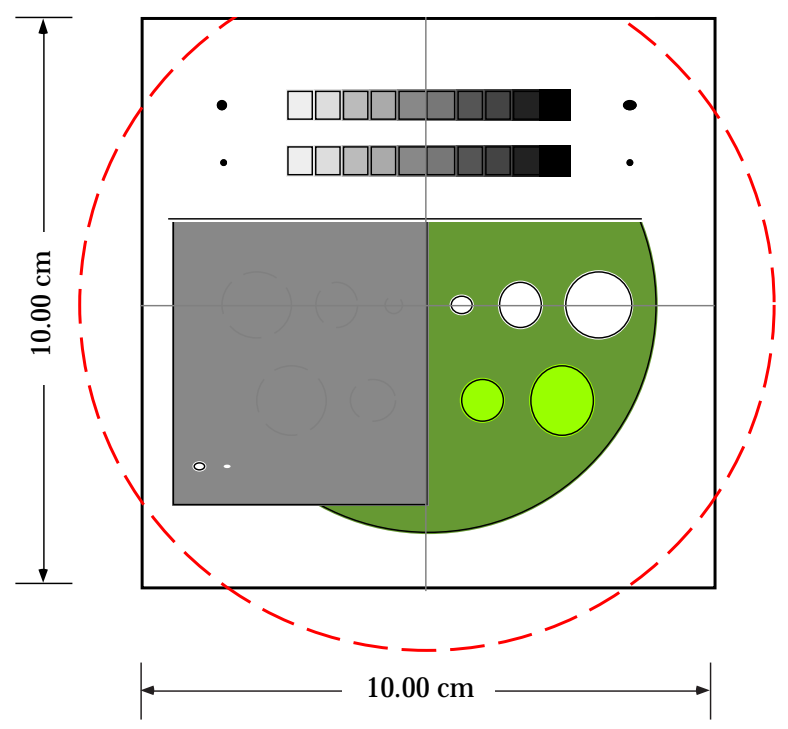

COG Image (simulation)

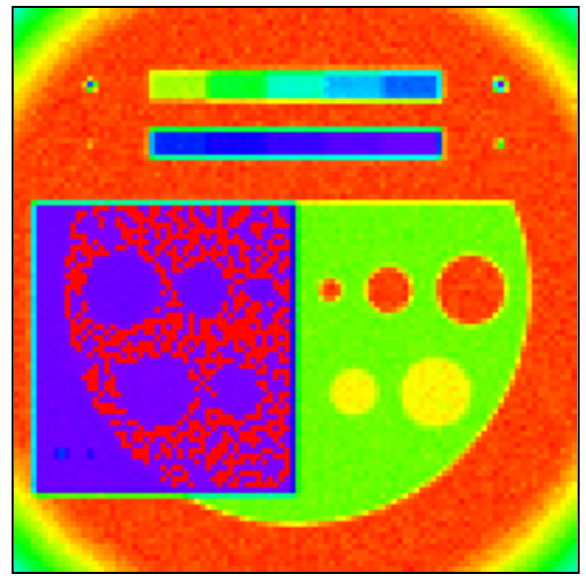

Figure 6. Thick target with simulated defects, and the COG simulation of its $14 \mathrm{MeV}$ neutron radiograph. Hole "defects" in low-Z material are resolved.

\section{SUMMARY}

The COG multiparticle radiation transport code, deployed in a parallel computation over many CPUs, can produce highly resolved and realistic simulations of neutron and photon radiographs of thick objects. The code accurately models all the physical processes which the radiation traversing the object undergoes, and can reliably separate out the image contributions due to, for example, primary (direct) radiation, once or twice scattered radiation, and Bremsstrahlung photons. COG can also readily assess the performance of radiation-based contraband detection systems. COG allows the parameter space of a particular design to be fully explored before the designer commits to a hardware realization.

\section{ACKNOWLEDGMENTS}

This work was performed at the University of California, Lawrence Livermore National Laboratory under the auspices of the U.S Department of Energy (contract \# W-7405-Eng-48). 


\section{REFERENCES}

1. T. Wilcox and E. Lent, COG - A Particle Transport Code Designed to Solve the Boltzmann Equation for DeepPenetration (Shielding) Problems, Vol. 1 Users Manual, LLNL Rept. \# M-221-1 (1989); see also R. Buck and E. Lent, "COG: A New, High-Resolution Code for Modeling Radiation Transport," LLNL Energy and Technology Review (June 1993); additional information on COG may be obtained via the Web at URL http://wwwphys.llnl.gov/N_Div/COG/ .

2. W. Nelson, H. Hirayama, and D. Rogers, The EGS4 Code System, Stanford Linear Accelerator Center, Stanford University, Stanford, CA, Rept. 265 (December 1985).

3. T. Wilcox and E. Lent, COG - A Particle Transport Code Designed to Solve the Boltzmann Equation for DeepPenetration (Shielding) Problems, Vol. 4: Benchmark Problems, LLNL Rept. \# M-221-4 (1989).

4. A. Gavron, High Energy Neutron Radiography - Observing the LiD, P-23 (Los Alamos National Laboratory) memorandum written on behalf of A. Bridge, C. Morris, M. Murray, S. Seestrom, J. Ullmann, W. Abfalterer, and A. Gavron of LANL and F. Dietrich and T. Wenaus of LLNL (December 7, 1994) 